\title{
PtyNAMi: ptychographic nano- analytical microscope at PETRA III: interferometrically tracking positions for 3D x-ray scanning microscopy using a ball-lens retroreflector
}

Christian G. Schroer, Martin Seyrich, Maik Kahnt, Stephan Botta, Ralph Döhrmann, et al.

Christian G. Schroer, Martin Seyrich, Maik Kahnt, Stephan Botta, Ralph Döhrmann, Gerald Falkenberg, Jan Garrevoet, Mikhail Lyubomirskiy, Maria Scholz, Andreas Schropp, Felix Wittwer, "PtyNAMi: ptychographic nanoanalytical microscope at PETRA III: interferometrically tracking positions for 3D x-ray scanning microscopy using a ball-lens retroreflector," Proc. SPIE 10389, X-Ray Nanoimaging: Instruments and Methods III, 103890E (7 September 2017); doi: 10.1117/12.2273710

Event: SPIE Optical Engineering + Applications, 2017, San Diego, California, United States 


\title{
PtyNAMi: Ptychographic nano-analytical microscope at PETRA III - interferometrically tracking positions for 3D x-ray scanning microscopy using a ball-lens retroreflector
}

\author{
Christian G. Schroer ${ }^{\mathrm{a}, \mathrm{b}}$, Martin Seyrich ${ }^{\mathrm{a}, \mathrm{b}}$, Maik Kahnta,b, Stephan Botta ${ }^{\mathrm{a}}$, Ralph Döhrmann ${ }^{\mathrm{a}}$, \\ Gerald Falkenberg $^{\mathrm{a}}$, Jan Garrevoet ${ }^{\mathrm{a}}$, Mikhail Lyubomirskiy ${ }^{\mathrm{a}}$, Maria Scholz ${ }^{\mathrm{a}, \mathrm{b}}$, \\ Andreas Schropp ${ }^{\mathrm{a}}$, and Felix Wittwer ${ }^{\mathrm{a}, \mathrm{b}}$ \\ ${ }^{a}$ Deutsches Elektronen-Synchrotron (DESY), Notkestr. 85, 22607 Hamburg, Germany \\ ${ }^{b}$ Department Physik, Universität Hamburg, Luruper Chaussee 149, 22761 Hamburg, Germany
}

\begin{abstract}
In recent years, ptychography has revolutionized x-ray microscopy in that it is able to overcome the diffraction limit of x-ray optics, pushing the spatial resolution limit down to a few nanometers. However, due to the weak interaction of $\mathrm{x}$ rays with matter, the detection of small features inside a sample requires a high coherent fluence on the sample, a high degree of mechanical stability, and a low background signal from the x-ray microscope. The x-ray scanning microscope PtyNAMi at PETRA III is designed for high-spatial-resolution 3D imaging with high sensitivity. The design concept is presented with a special focus on real-time metrology of the sample position during tomographic scanning microscopy.
\end{abstract}

Keywords: X-ray scanning microscopy, ptychography, nanopositioning, interferometry

\section{INTRODUCTION \& GENERAL DESIGN CONSIDERATIONS}

Hard x-ray scanning microscopy is an ideal tool to follow physical and chemical processes in complex matter and thus finds applications in many fields of science, such as chemistry and physics, the bio-medical, materials, geo and environmental sciences, as well as in nanotechnology. The large penetration depth of $\mathrm{x}$ rays in matter allows one to study the inner structures of an object or to follow physical or chemical processes in-situ or under working conditions (operando) inside special sample environments, e. g., a catalytic reactor ${ }^{1-4}$ or a pressure cell. Various x-ray analytical techniques can be used as contrasts in the scanning microscope, allowing for quantitative measurements of physical and chemical properties inside the sample. For instance, x-ray fluorescence, x-ray scattering, and x-ray absorption yield the chemical composition (with trace element sensitivity), the local atomic and mesoscopic structure, and the local chemical environment of an element of interest, respectively. In combination with tomography, scanning microscopy yields local quantitative measurements of these physical and chemical quantities in three dimensions inside a sample without destructive preparation or inside a special environment. ${ }^{5-9}$

In x-ray scanning microscopy, the sample is raster scanned through a confined beam, measuring at each position of the scan one or more of the above-mentioned x-ray analytical techniques. The spatial resolution in this setting is limited by the lateral size of the x-ray beam. The smallest beams for given optics are obtained from diffraction-limited focusing, i. e., when the aperture of the optics is illuminated coherently. The smallest beam size is then determined by the numerical aperture of the optics, provided aberrations are negligible. Today, there is a variety of x-ray optics available for scanning microscopy based on external total reflection, diffraction, and refraction. As only the coherent flux from the source can be focused to the diffraction limit, the source needs to have high spectral brightness. Therefore, x-ray microscopy is mainly limited to modern synchrotron radiation sources, such as the European Synchrotron Radiation Facility (ESRF) in Grenoble, France, the Advanced Photon Source (APS) near Chicago, USA, SPring-8 in Japan, or PETRA III at DESY in Hamburg,

Further author information: (Send correspondence to Christian G. Schroer)

Christian G. Schroer: E-mail: christian.schroer@desy.de, Telephone: +49 4089982503.

X-Ray Nanoimaging: Instruments and Methods III, edited by Barry Lai, Andrea Somogyi, Proc. of SPIE Vol. 10389, 103890E · C 2017 SPIE CCC code: $0277-786 X / 17 / \$ 18 \cdot$ doi: $10.1117 / 12.2273710$ 
Germany. This technique will benefit in particular from recent developments in storage-ring technology, i. e., the so-called multi-bend achromat technology pioneered by MAX-IV in Lund, Sweden. It can significantly reduce the (horizontal) emittance of a storage ring compared to conventional storage-ring designs and thus boost the spectral brightness. Today, most of the synchrotron radiation sources worldwide, including PETRA III at DESY, have plans to upgrade the storage ring to this new technology.

In the hard x-ray range, the numerical aperture $(N A)$ of $\mathrm{x}$-ray optics is typically in the range of $10^{-3}$ and thus the lateral Airy disc size of x-ray beam is in the range of a few tens of nanometers. The size of the Airy disc is given by Abbe's resolution formula

$$
d_{t}=\alpha \frac{\lambda}{2 N A}
$$

where $\alpha$ is a number close to unity that depends on the aperture function, $\lambda$ is the x-ray wavelength. Recent advances in $\mathrm{x}$-ray optics have pushed these limits to the single-digit nanometer range, ${ }^{10,11}$ and theoretical considerations predict focusing in the (sub-)nanometer range. ${ }^{12,13}$ Improving the numerical aperture to these physical limits is very challenging and currently limited by technology.

The limitation in spatial resolution given by today's x-ray optics can be overcome by coherent x-ray diffraction imaging techniques, such as scanning coherent diffraction microscopy also called ptychography. ${ }^{14,15}$ In recent years this technique has developed into a routine x-ray microscopy technique available at various synchrotron radiation sources, including the hard x-ray nanoprobe station of beamline P06 at PETRA III. ${ }^{16,17}$ In this scanning microscopy technique, a coherent far-field diffraction pattern of the illuminated region on the sample is recorded at each scan point. From these data and the knowledge of their relative scanning positions, the complex transmission function (integral of the complex refractive index along the beam direction) can be reconstructed together with the complex wave field at the sample position using phase retrieval algorithms. ${ }^{18,19}$ The spatial resolution of these reconstructions is not limited by the numerical aperture of the optics but determined by the maximal momentum transfer in the diffraction patterns and can significantly exceed the resolution of conventional scanning microscopy.

\section{HARD X-RAY NANOPROBE STATION AT PETRA III}

The X-ray scanning microscope (nanoprobe) ${ }^{16,17}$ at beamline P06 was commissioned in 2011 and went into user operation at the beginning of 2012. It was designed based on nanofocusing refractive x-ray lenses (NFLs) ${ }^{20,21}$ and is located at about $98 \mathrm{~m}$ from the source. The transverse coherence length of the beam is matched to the aperture by a set of refractive x-ray lenses made of beryllium located at $43.5 \mathrm{~m}$ from the source. ${ }^{17}$ They create a (sometimes virtual) secondary source along the beamline and allow various modes of operation: ${ }^{16}$

- A high-coherence mode, for which the lateral coherence length is made much larger than the aperture of the nanofocusing optics. In this mode, a high lateral coherence is achieved at the expense of flux.

- Matching the lateral coherence length to the size of the aperture of the nanofocusing optics to reach highest flux at smallest focus size (highest flux density).

- A high-flux mode, for which the lateral coherence length is made smaller than the aperture of the nanofocusing optics. In this mode, the focus is no longer diffraction limited, but a part of the x-ray beam that is larger than the coherent fraction is focused.

The scanner unit was designed to scan the sample in three translations (along and perpendicular to the optical axis) and one rotation (around the vertical axis) through the beam focused by nanofocusing refractive x-ray lenses. ${ }^{20,21}$ Two such lenses that each focus the beam in one direction need to be aligned in crossed geometry to focus the $\mathrm{x}$ rays to a common focal plane (requiring a minimum of ten degrees of freedom). A pair of slits defines the entrance aperture of the optics and a pinhole behind the lenses cleans the focused beam from scattered radiation. ${ }^{17}$ As the imaging geometries and the requirements for their alignment are very similar for Fresnel zone plates (FZP) and multi-layer Laue lenses (MLL), these optics can also be used in the scanning microscope. ${ }^{22-25}$

In order to exploit the various x-ray analytical contrasts, a series of different detectors are employed: an energy dispersive detector (silicon drift diode, Vortex-EM from Hitatchi), various photon-counting pixel detectors 

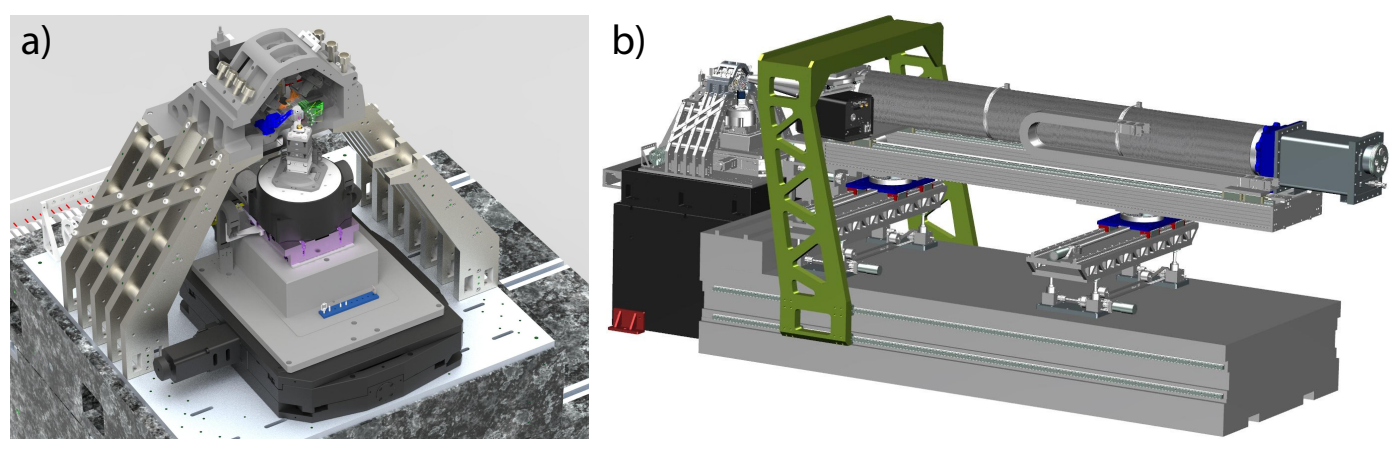

Figure 1. PtyNAMi at beamline P06 at PETRA III: a) Scanner unit (in operation since beginning of 2017) and b) overview including the detector table with flight-tube system and integrated windowless in-vacuum pixel detector (Eiger $\mathrm{X} 4 \mathrm{M})$.

for SAXS and WAXS measurements (Pilatus 300k from Dectris, Lambda 750k from X-Spectrum, and since recently an Eiger X 4M from Dectris), a high-resolution x-ray camera (Optique-Peter microscope combined with a PCO.4000 from PCO), and a calibrated PIN-Diode (Hamamatsu). While the energy dispersive detector is located on a separate table and points at the sample under $90^{\circ}$ from the side, all other detectors are placed on a large detector table behind the scanner. ${ }^{17}$ An evacuated tube can be used to reduce the air scattering in SAXS and high-resolution WAXS geometry.*

With the instrument, a series of experiments was carried out, reaching from the verification of the structural integrity of polymer solar cells, ${ }^{28}$ catalysis, ${ }^{4}$ to the investigation of semi-conductor nanorods. ${ }^{26,27,29-31}$ The instrument served as a test bench for developments of methods and instrumentation in x-ray microscopy, pushing ptychography to high-resolution ${ }^{15}$ and sensitivity ${ }^{32}$ and combining the technique with resonant scattering to obtain chemical contrast. ${ }^{33}$ Ptychography was used to characterize various x-ray optics, including refractive optics like adiabatically focusing lenses (AFL) ${ }^{34,35}$ refractive lamellar lenses (RLL), ${ }^{36}$ and kinoform lenses, ${ }^{24}$ and diffractive optics, such as multilayer Laue lenses (MLL) $)^{22,25}$ and Fresnel zone plates (FZP). ${ }^{23}$ To this end, optics characterization schemes ${ }^{37,38}$ and a way to design corrective phase plates for x-ray optics were developed. ${ }^{39}$ Based on these developments, the new Ptychographic Nano-Analytical Microscope (PtyNAMi) was designed that is described in more detail below.

\section{PTYNAMI: THE PTYCHOGRAPHIC NANO-ANALYTICAL MICROSCOPE AT PETRA III}

The spatial resolution in ptychography is limited by the photon statistics in the diffraction patterns and by various sources of deviations from the physical model underlying the ptychographic reconstruction. Deviations from the ptychographic model can include background scattering from the scanning microscope, mechanical instabilities and drifts as well as partial coherence in the illumination. While these deviations from the ideal ptychographic model can be included in the model, best imaging results are obtained when these deviations are mitigated instrumentally. This is the rationale behind improving the hard x-ray scanning microscope (nanoprobe) at beamline P06 at PETRA III after four years of user operation. The new Ptychographic Nano-Analytical Microscope (PtyNAMi) was designed to address the above-mentioned issues:

- capturing a larger coherent fraction by appropriate prefocusing and matching the coherence length to the aperture of the nanofocusing optics.

- improving and tracking the mechanical stability of optics relative to the sample scanner.

*In high-resolution WAXS geometry, a photon-counting pixel detector is placed at a large distance from the sample,

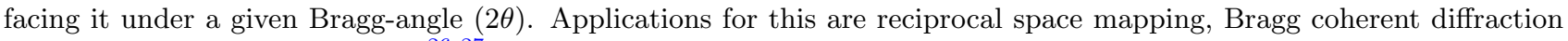
imaging, or Bragg ptychography. ${ }^{26,27}$ 
- reducing the scattering background of the instrument by an appropriate flight tube integrating a windowless in-vacuum pixel detector (Eiger X 4M).

Fig. 1 shows the general design of the new scanning microscope. The optics unit [Fig. 1(a)] was installed and commissioned at PETRA III during the winter shutdown and is in user operation since the first run of 2017. The Eiger X 4M has been commissioned at the end of 2016 and is in user operation since the beginning of 2017. The new flight-tube system [cf. Fig. 1(b)] that will allow for windowless in-vacuum operation of the detector is planned to be installed in 2018, as well as a new and more stable sample scanner.

Mechanical stability and the accurate metrology of sample and optics are crucial for the scanning microscope. In this article, we describe a versatile laser-interferometer system used to track the sample in the two directions transverse to the optical axis.

\section{MEASURING THE SAMPLE POSITION IN SCANNING TOMOGRAPHY}

For scanning microscopy and tomography it is essential to know where the beam hits the sample. Position uncertainties can arise from vibrations of the focusing optics and of the sample. For data analysis, only the relative movement of these objects is required, yet we decided to measure the positions of both optics and sample with regard to a common reference point on the frame of the optics unit of the scanner. In PtyNAMi, the sample is scanned through the nanobeam, while the optics are kept fixed (no movement of stages) after initial alignment. While measuring the optics' position is relatively straight forward, keeping track of the sample position within tomographic scans is more challenging and will be described in the following.

All position measurements are made by laser-interferometry. The interferometers are commercial systems provided by SmarAct and attocube systems using a modulated diode laser operated at a wavelength around $\lambda=1550 \mathrm{~nm}$. The systems use the principle of homodyne phase quadrature, i. e., the demodulated interference signal provides the sine and cosine of the displacement. These functions draw an ellipsis in a 2D plane, the position on which is proportional to the displacement. The commercial system consists of a controller unit comprising the diode laser and an FPGA for signal evaluation and optical fibers terminating in interferometer heads. These heads have a beamsplitter in their tip which provides the reference signal for the interference.

To measure the position of the sample, we followed the following design principles: The reflector used for measuring the sample's position should be placed closely to the sample so that the reflector's motion is representative of the sample's motion (small Abbe errors). However, space around the sample is restricted from several directions: the sample is mounted on the sample stage below, the focusing optics and a cleaning aperture are placed in close proximity upstream of the sample, a fluorescence detector is placed on the right-hand side, and the downstream direction needs to stay clear for SAXS and WAXS measurements. Therefore, the interferometer access is only possible from the side of the sample opposing the fluorescence detector. We decided to place a ball lens retroreflector below the sample, with three interferometers mounted in an arc pointing into it (cf. Fig. 2).

Ball lenses are glass spheres with homogeneous refractive index $n$. Their focal length $f$ can be calculated in ray optics by treating them as a thick lens: ${ }^{40}$

$$
f=\frac{n r}{2(n-1)},
$$

where $r$ is the radius of the sphere and the surrounding medium is of refractive index 1 . The principal planes lie at the center of the lens. For $n=2$ the focal length $f$ is equal to the radius $r$. Collimated incoming light is therefore focused onto the opposite side of the sphere and efficiently retroreflected with similar collimation. This makes the ball lens an effective retroreflector from all perspectives, provided the center of the sphere lies on the optical axis and the beam does not extend beyond the paraxial regime of the lens (spherical approximation). In particular, the ball can be rotated over more than $180^{\circ}$ while continuously maintaining its ability to retroreflect the beam. This means that the position of the sample can be continuously tracked during a tomographic scan allowing easy alignment of the projections. In addition, the relative positions can be monitored during rocking curve scans of the sample in Bragg geometry. 
a)

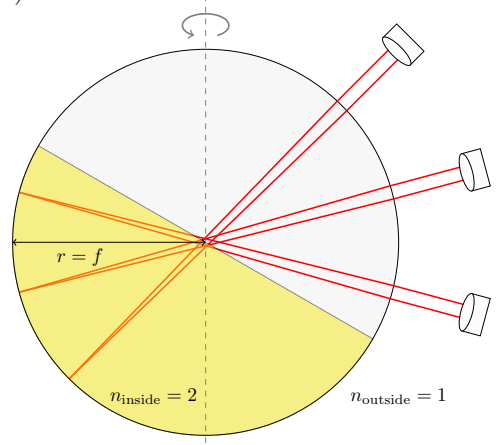

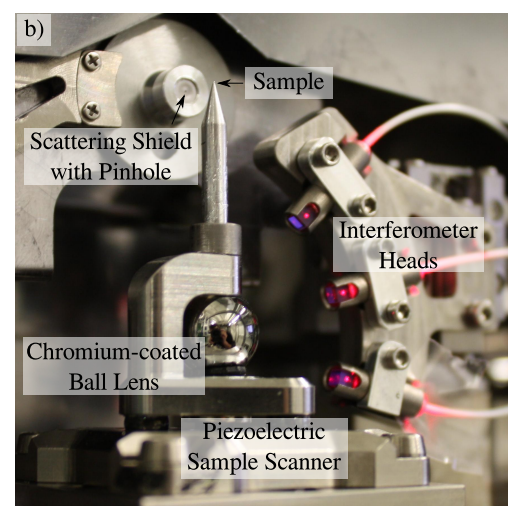

c)

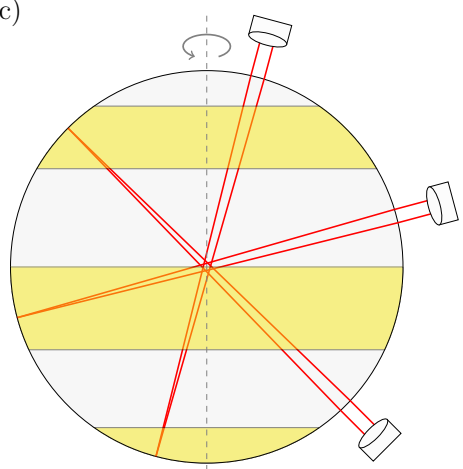

Figure 2. a) Schematic optical setup of the laser interferometers to measure the location of the sample in the two directions transverse to the x-ray beam. Three laser interferometers are used to triangulate the position of the center of a ball lens. The ball lens is plated with chromium (depicted here in yellow) on one side for better reflectivity. b) photograph of the sample region in the scanning microscope, showing the practical realization of the scheme shown in a). c) Alternative triangulation geometry that maximizes the precision and accuracy of the measurement as well as possible rotation angles.

To track the sample position, we use three laser interferometers pointing at the ball lens in the plane perpendicular to the x-ray optical axis and under different angles that are approximately $30^{\circ}$ apart. ${ }^{\dagger}$ From the three distances measured, the position of the ball lens can be triangulated. Measuring three distances in the plane introduces some redundancy, allowing to improve the precision and accuracy of the position measurement but also to make up for the potential loss of one of the interferometer signals during a scan.

Metal coatings can be used to enhance the reflectivity of the lens. A thick coating of one hemisphere optimizes reflectivity, but limits the acceptance of the retroreflector to the other hemisphere. Depending on the rotation of the ball lens relative to the interferometers, the coating can block the signal of the lowest interferometer from certain angles (cf. Fig. 2). Currently, we are using the setup shown in Fig. 2a). In tomographic scans over the full $180^{\circ}$, the signal from the lowest interferometer is lost at both ends of the angular range. Due to the redundancy of the interferometers, the position can still be tracked in these cases with a slight loss in precision. This geometry illustrated in Fig. 2a),b) has proven most reliable and is currently used in the scanning microscope.

In applications that require a wider range of rotation the coating scheme can be adapted. One possibility is to coat the entire ball lens homogeneously with a thin semitransparent layer with an optimal reflectivity of 0.33. In this case, the overall reflectivity of the lens, i. e., transmission into the lens, reflection at the back side of the ball lens and transmission into air/vacuum, is $R=0.148$. This reflectivity is uniform from all perspectives over $4 \pi$ solid angle. Applying this coating evenly over the entire sphere with the ideal thickness was found to be challenging, furthermore the reduced reflectivity reduces the signal strength in the interferometer, hence requiring more accurate alignment. These limitations deemed this coating variant impractical for beamline operation.

A third option comprises a ball lens coated with horizontal stripes [cf. Fig. 2c)]. This permits to reflect beams with full signal strength while rotating $360^{\circ}$ around the vertical axis, e. g., for tomography. Furthermore, the directions of observations of the interferometers can be tilted $60^{\circ}$ apart from each other, which is the optimal configuration of three observation axes in a plane to achieve uniform measurement uncertainties. This latter scheme has not been tested so far, due to difficulties in fabricating the more complex metallization pattern onto a ball lens.

In the setup [cf. Fig. 2b)] a ball lens with a diameter of $1 \mathrm{~cm}$ fabricated from Ohara S-LAH79 with a refractive index of $n=1.95518$ is used. The lower hemisphere is coated with chromium, the equator between coated and uncoated hemispheres is tilted $30^{\circ}$ with respect to the horizontal plane. The interferometer axes observe the motion from $-15^{\circ}, 15^{\circ}$ and $45^{\circ}$ with respect to the horizontal plane as shown in Fig. 2a),b). The arc mounting

${ }^{\dagger}$ The position along the beam is not tracked by interferometers, as x-ray scanning microscopy is rather insensitive to small (sub-micrometer) position errors along the optical axis due to the large depth of focus of x-ray optics. 

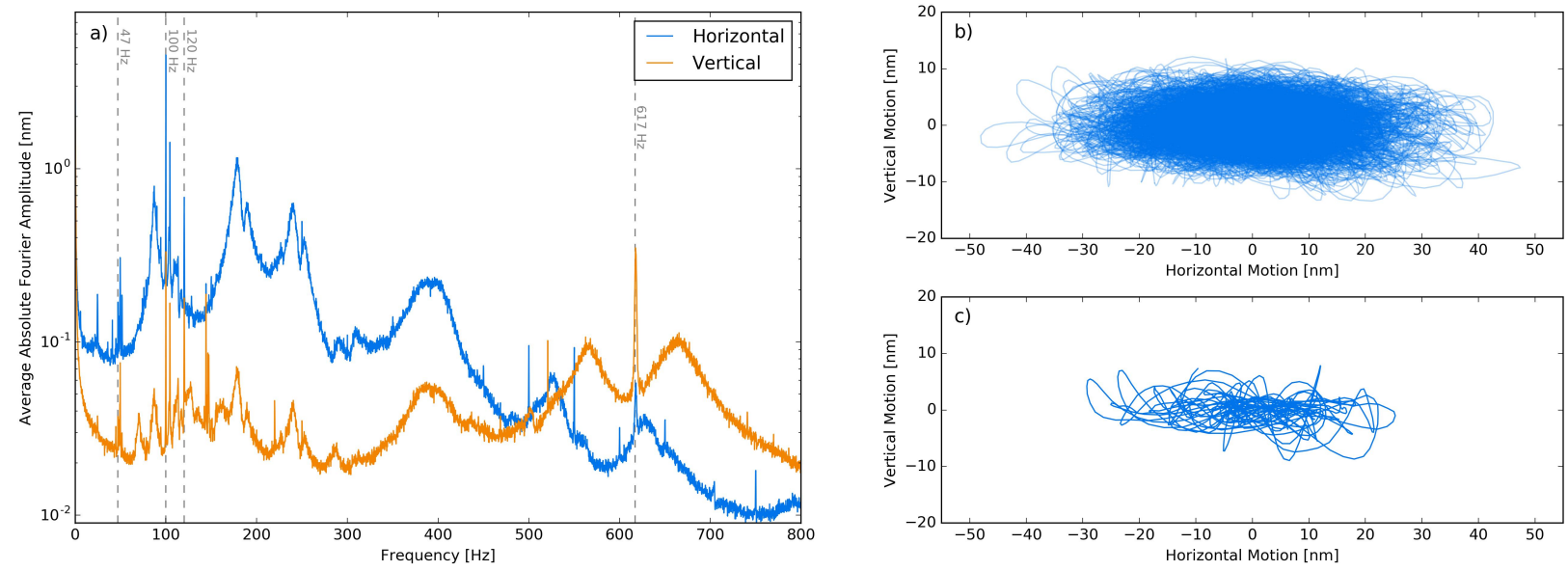

Figure 3. a) Spectrum of horizontal and vertical motion during a $4.75 \mathrm{~s}$ exposure of a step scan. Fourier amplitudes of the position time series at 121 scan points were averaged. b) Trace of the sample during a single $4.75 \mathrm{~s}$ exposure. c) An excerpt of the trace in b) shows how the sample moved within $100 \mathrm{~ms}$.

the interferometer heads is motorized with three linear piezo translation stages for alignment to the sample position.

\section{CHARACTERIZATION OF MOVEMENT}

Using the interferometric setup described in section 4 and shown in Fig. 2, we have studied the stability of the former scanner of the nanoprobe. In this study, we verified that the setup was suitable to track the motion of the sample over time scales from sub-milliseconds to hours.

\subsection{Tracking Fast Sample Movements: Vibrations}

The sampling rates of the interferometers can reach into the megahertz range. This permits to follow the movement of the sample due to vibrations on all relevant time scales and analyze the characteristic spectrum of the motion. Typical sampling rates are set between $1 \mathrm{kHz}$ and $10 \mathrm{kHz}$. Vibration spectra were correlated with (unwanted) sources of vibration in and outside of the experiment. Vibration measurements of various components were done using either additional interferometers or seismometers. (The latter devices measure accelerations instead of relative displacements.)

For a demonstration we scanned an area of $1 \times 1 \mathrm{~mm}^{2}$ in $10 \times 10$ steps. At each scan point a time series of the positions was recorded during a dwell time of $4.75 \mathrm{~s}$. Figure 3a) shows the resulting spectrum averaged over all scan points. The most prominent peak is at $100 \mathrm{~Hz}$ sharp, indicating a vibration source powered by a rectified alternating current at $50 \mathrm{~Hz}$. Indeed, the peak could be attributed in parts to an electromagnet used in the proximity of the setup. The same explanation is reasonable for the $50 \mathrm{~Hz}$ peak. The peak at $47 \mathrm{~Hz}$ was caused by a vacuum pump, the $120.2 \mathrm{~Hz}$ peak is the eigenfrequency of the granite table. Another prominent peak around $617 \mathrm{~Hz}$ was caused by the air-bearing rotation stage on which the sample is mounted.

Figures $3 \mathrm{~b}$ ) and c) show the trace of the sample motion at rest at a given scan point, i. e,. without intentional movement of the scanner. The figures clearly show that the interferometer can finely sample the trajectory of the sample. The trace fills an ellipse of about $70 \mathrm{~nm} \times 20 \mathrm{~nm}$ (peak to peak). On average, fifty percent of the positions lie inside a rectangular range of $14.8 \mathrm{~nm} \times 4.6 \mathrm{~nm}$ in horizontal and vertical direction, respectively. The average standard deviations of the distributions are $10.9 \mathrm{~nm}$ (horizontal) by $3.2 \mathrm{~nm}$ (vertical).

These diagnostics were very helpful to identify sources of vibration in the noisy experimental environment of a synchrotron radiation source. This also helps to identify unusual sources of vibrations, such as construction work. In addition, it was demonstrated that the vibrations during a scan can be followed in real time, giving access to sample positions during each individual exposure. This allows to better model the individual point spread in 

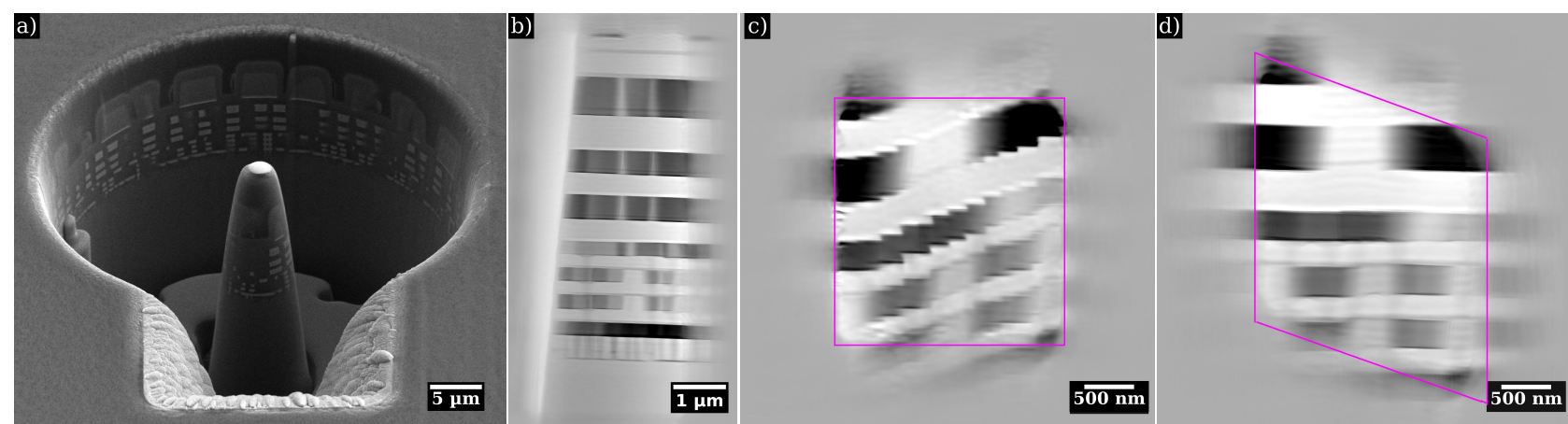

Figure 4. Ptychography experiment on microchip test structure. a) SEM image taken during sample preparation. The central cone is used as the sample in the x-ray experiment. b) Reconstructed phase shift of the sample without perceivable drift. c) Reconstructed phase shift of sample from a scan with significant vertical drift. This reconstruction used encoder positions of the scanner. d) Corrected reconstruction of c) using interferometer position values instead of those from the encoder. All ptychographic reconstructions used local algorithmic position correction. ${ }^{41}$ Between b) and c,d) the sample was rotated by $16^{\circ}$.

a conventional scanning micrograph or to assess the degree of coherence in a diffraction pattern recorded in a ptychographic scan.

The parameters extracted from the scan above demonstrate that the previous x-ray microscopy scanner was sufficiently stable for conventional scanning microscopy with a resolution level from 50 to $100 \mathrm{~nm}$. However, for ptychographic microscopy that pushes the spatial resolution into the sub-10 $\mathrm{nm}$ regime, significantly higher mechanical stability is required. Therefore, the sample scanner of the new PtyNAMi is completely redesigned and will be installed in 2018 .

\subsection{Tracking Slow Sample Movements: Drifts}

Temperature changes in the experimental hutch can lead to thermal expansion of microscope components. The nanoprobe at P06 is temperature-stable to $10 \mathrm{mK}$ when not being accessed, but any in-person interaction, especially changing the sample, can lead to hour-long drifts. These drifts are difficult to detect since they are not captured by the stages' encoders and cause a distorted imaging result. We demonstrate the correction capabilities for this kind of error using interferometry with the following example.

The sample was a conical piece of a microchip test structure. An SEM image of the sample during the preparation process is shown in Fig. 4a). $\mathrm{X}$ rays at $16 \mathrm{keV}$ were focused with nanofocusing refractive lenses with a working distance of $30 \mathrm{~mm}$. The full width at half maximum of the focused beam was measured to be $96 \times 99 \mathrm{~nm}^{2}$. Diffraction patterns were recorded at $2.1 \mathrm{~m}$ distance from the sample using an Eiger X $4 \mathrm{M}$ in air.

Figure $4 \mathrm{~b}$ ) shows the ptychographic reconstruction of a $2.5 \times 7 \mathrm{\mu m}^{2}$ area on the edge of the conical sample. This area was sampled in $50 \times 140$ steps with an exposure time of $0.3 \mathrm{~s}$ per diffraction pattern. The measurement was done under stable experimental conditions.

The quality of the micrographs changes significantly if a vertical sample drift of about $1.7 \mu \mathrm{m} \mathrm{h}^{-1}$ occurs as in the following example. We scanned a smaller area of $2 \times 2 \mu^{2}$ in $40 \times 40$ steps with an exposure time of $0.5 \mathrm{~s}$ each. Two ptychographic reconstructions of the sample phase shift can be seen in Fig. 4c) and d). In these figures the scanned area is marked in magenta.

Figure 4c) has been reconstructed using the encoder data of the piezoelectric sample scanner. The reconstruction exhibits a staircase-shaped structure. The recorded diffraction patterns clearly indicate strong horizontal edges in the sample, but due to the drifting of the sample mainly in vertical direction the ptychgraphic algorithm tries to accommodate this drift into a false rectangular field of view, as suggested by the encoder position values. These two conflicting inputs cause a mismatch in the ptychographic reconstruction algorithm, which gives rise to the staircase artifact seen in Fig. 4c). 
In contrast, Fig. 4d) was reconstructed using the very same algorithm and identical diffraction patterns, but with positions measured by the interferometers. In this case, the field of view is distorted by the vertical drift, but the structures of the sample are reconstructed correctly. This shows how interferometric position monitoring can compensate unintentional sample motion under imperfect experimental conditions.

\section{CONCLUSION AND OUTLOOK}

PtyNAMi is the next generation hard x-ray scanning microscope at beamline P06 of PETRA III at DESY in Hamburg. The x-ray optics unit together with the laser interferometers for position control of the sample have been commissioned. Based on the experience gained with the previous design, the scanning microscope is improved in terms of mechanical stability and reduced background. The new sample scanner designed for maximal stability as well as the new detector system designed to reduce background signals will be completed in 2018. In this article, we presented an interferometric positioning system for the sample, which allows tracking the sample position relative to the optics in scanning microscopy and tomography on all relevant time scales to track vibrations and long term drifts. The interferometer system will be crucial for high-resolution scanning $\mathrm{x}$-ray microscopy in the noisy environment of a synchrotron radiation source in user operation.

\section{ACKNOWLEDGEMENTS}

We thank Thomas Keller and Satishkumar Kulkarni from DESY NanoLab for their help with the sample preparation of the microchip test structure that was provided by Infineon Technologies, Dresden. This work was supported by the German Ministry of Education and Research (BMBF) under Grant No. 05K13OD4 and by VH-VI-403 of the Impuls- und Vernetzungsfonds (IVF) of the Helmholtz Association of German Research Centres. Beamtime at beamline P06 at PETRA III was granted within the user program of DESY, a member of the Helmholtz-Association.

\section{REFERENCES}

[1] Grunwaldt, J.-D., Hannemann, S., Schroer, C. G., and Baiker, A., "2D-mapping of the catalyst structure inside a catalytic microreactor at work: Partial oxidation of methane over $\mathrm{Rh} / \mathrm{Al}_{2} \mathrm{O}_{3}$, , J. Phys. Chem. $B$ 110, 8674-8680 (2006).

[2] Grunwaldt, J.-D. and Schroer, C. G., "Hard and soft x-ray microscopy and tomography in catalysis: Bridging the different time and length scales," Chem. Soc. Rev. 39, 4741 (2010).

[3] Baier, S., Damsgaard, C. D., Scholz, M., Benzi, F., Rochet, A., Hoppe, R., Scherer, T., Shi, J., Wittstock, A., Weinhausen, B., Wagner, J. B., Schroer, C. G., and Grunwaldt, J.-D., "In situ ptychography of heterogeneous catalysts using hard x-rays: High resolution imaging at ambient pressure and elevated temperature," Microscopy and Microanalysis 22(1), 178-188 (2016).

[4] Baier, S., Damsgaard, C. D., Klumpp, M., Reinhardt, J., Sheppard, T., Balogh, Z., Kasama, T., Benzi, F., Wagner, J. B., Schwieger, W., Schroer, C. G., and Grunwaldt, J.-D., "Stability of a bifunctional Cubased corezeolite shell catalyst for dimethyl ether synthesis under redox conditions studied by environmental transmission electron microscopy and In Situ x-ray ptychography," Microscopy and Microanalysis 23, 501512 (2017). doi:10.1017/S1431927617000332.

[5] Simionovici, A. S., Chukalina, M., Schroer, C., Drakopoulos, M., Snigirev, A., Snigireva, I., Lengeler, B., Janssens, K., and Adams, F., "High-resolution x-ray fluorescence microtomography of homogeneous samples," IEEE Trans. Nucl. Sci. 47(6), 2736-2740 (2000).

[6] Schroer, C. G., "Reconstructing x-ray fluorescence microtomograms," Appl. Phys. Lett. 79(12), 1912-1914 (2001).

[7] Schroer, C. G., Benner, B., Günzler, T. F., Kuhlmann, M., Lengeler, B., Schröder, W. H., Kuhn, A. J., Simionovici, A. S., Snigirev, A., and Snigireva, I., "High resolution element mapping inside biological samples using fluorescence microtomography," in [Developments in X-Ray Tomography III], Bonse, U., ed., Proceedings of the SPIE 4503, 230-239 (2002). 
[8] Schroer, C. G., Kuhlmann, M., Günzler, T. F., Lengeler, B., Richwin, M., Griesebock, B., LützenkirchenHecht, D., Frahm, R., Ziegler, E., Mashayekhi, A., Haeffner, D., Grunwaldt, J.-D., and Baiker, A., "Mapping the chemical states of an element inside a sample using tomographic x-ray absorption spectroscopy," Appl. Phys. Lett. 82(19), 3360-3362 (2003).

[9] Schroer, C. G., Kuhlmann, M., Roth, S. V., Gehrke, R., Stribeck, N., Almendarez-Camarillo, A., and Lengeler, B., "Mapping the local nanostructure inside a specimen by tomographic small angle x-ray scattering," Appl. Phys. Lett. 88(16), 164102 (2006).

[10] Mimura, H., Handa, S., Kimura, T., Yumoto, H., Yamakawa, D., Yokoyama, H., Matsuyama, S., Inagaki, K., Yamamura, K., Sano, Y., Tamasaku, K., Nishino, Y., Yabashi, M., Ishikawa, T., and Yamauchi, K., "Breaking the $10 \mathrm{~nm}$ barrier in hard-X-ray focusing," Nature Physics 6, 122-125 (2010).

[11] Morgan, A. J., Prasciolu, M., Andrejczuk, A., Krzywinski, J., Meents, A., Pennicard, D., Graafsma, H., Barty, A., Bean, R. J., Barthelmess, M., Oberthuer, D., Yefanov, O., Aquila, A., Chapman, H. N., and Bajt, S., "High numerical aperture multilayer Laue lenses," Scientific Reports 5, 09892 (2015).

[12] Schroer, C. G., "Focusing hard x rays to nanometer dimensions using Fresnel zone plates," Phys. Rev. B 74, 033405 (2006).

[13] Yan, H., Maser, J., Macrander, A., Shen, Q., Vogt, S., Stephenson, G. B., and Kang, H. C., "Takagi-taupin description of x-ray dynamical diffraction from diffractive optics with large numerical aperture," Phys. Rev. $B$ 76, 115438 (Sep 2007).

[14] Thibault, P., Dierolf, M., Menzel, A., Bunk, O., David, C., and Pfeiffer, F., "High-resolution scanning x-ray diffraction microscopy," Science 321(5887), 379-382 (2008).

[15] Schropp, A., Hoppe, R., Patommel, J., Samberg, D., Seiboth, F., Stephan, S., Wellenreuther, G., Falkenberg, G., and Schroer, C. G., "Hard x-ray scanning microscopy with coherent radiation: Beyond the resolution of conventional x-ray microscopes," Appl. Phys. Lett. 100, 253112 (2012).

[16] Schroer, C. G., Boye, P., Feldkamp, J. M., Patommel, J., Samberg, D., Schropp, A., Schwab, A., Stephan, S., Falkenberg, G., Wellenreuther, G., and Reimers, N., "Hard X-ray nanoprobe at beamline P06 at PETRA III," Nucl. Instrum. Meth. A 616(2-3), 93-97 (2010).

[17] Schroer, C. G., Baumbach, C., Döhrmann, R., Klare, S., Hoppe, R., Kahnt, M., Patommel, J., Reinhardt, J., Ritter, S., Samberg, D., Scholz, M., Schropp, A., Seiboth, F., Seyrich, M., Wittwer, F., and Falkenberg, G., "Hard x-ray nanoprobe of beamline P06 at PETRA III," AIP Conference Proceedings 1741, 030007 (2016).

[18] Maiden, A. M. and Rodenburg, J. M., "An improved ptychographical phase retrieval algorithm for diffractive imaging," Ultramicroscopy 109(10), 1256-1262 (2009).

[19] Thibault, P., Dierolf, M., Bunk, O., Menzel, A., and Pfeiffer, F., "Probe retrieval in ptychographic coherent diffractive imaging," Ultramicroscopy 109(4), 338-343 (2009).

[20] Schroer, C. G., Kuhlmann, M., Hunger, U. T., Günzler, T. F., Kurapova, O., Feste, S., Frehse, F., Lengeler, B., Drakopoulos, M., Somogyi, A., Simionovici, A. S., Snigirev, A., Snigireva, I., Schug, C., and Schröder, W. H., "Nanofocusing parabolic refractive x-ray lenses," Appl. Phys. Lett. 82(9), 1485-1487 (2003).

[21] Schroer, C. G., Kurapova, O., Patommel, J., Boye, P., Feldkamp, J., Lengeler, B., Burghammer, M., Riekel, C., Vincze, L., van der Hart, A., and Küchler, M., "Hard x-ray nanoprobe based on refractive x-ray lenses," Appl. Phys. Lett. 87(12), 124103 (2005).

[22] Braun, S., Kubec, A., Menzel, M., Niese, S., Krüger, P., Seiboth, F., Patommel, J., and Schroer, C., "Multilayer Laue lenses with focal length of $10 \mathrm{~mm}$," J. Phys. Conf. Ser. 425(5), 052019 (2012).

[23] Uhlén, F., Nilsson, D., Rahomäki, J., Belova, L., Schroer, C. G., Seiboth, F., Holmberg, A., Hertz, H. M., and Vogt, U., "Nanofabrication of tungsten zone plates with integrated platinum central stop for hard X-ray applications," Microelectronic Engineering 116, 40-43 (2014).

[24] Karvinen, P., Grolimund, D., Willimann, M., Meyer, B., Birri, M., Borca, C., Patommel, J., Wellenreuther, G., Falkenberg, G., Guizar-Sicairos, M., Menzel, A., and David, C., "Kinoform diffractive lenses for efficient nanofocusing of hard X-rays," Opt. Express 22(14), 16676-16685 (2014).

[25] Kubec, A., Braun, S., Niese, S., Krüger, P., Patommel, J., Hecker, M., Leson, A., and Schroer, C. G., "Ptychography with mulitlayer Laue lenses," J. Synchrotron Rad. 21, 1122-1127 (2014). 
[26] Stankevič, T., Hilner, E., Seiboth, F., Ciechonski, R., Vescovi, G., Kryliouk, O., Johansson, U., Samuelson, L., Wellenreuther, G., Falkenberg, G., Feidenhans'l, R., and Mikkelsen, A., "Fast strain mapping of nanowire light-emitting diodes using nanofocused x-ray beams," ACS nano 9(7), 6978 - 6984 (2015).

[27] Stankevič, T., Dzhigaev, D., Bi, Z., Rose, M., Shabalin, A., Reinhardt, J., Mikkelsen, A., Samuelson, L., Falkenberg, G., Vartanyants, I. A., and Feidenhans'l, R., "Strain mapping in an InGaN/GaN nanowire using a nano-focused x-ray beam," Appl. Phys. Lett. 107, 103101 (2015).

[28] Dam, H. F., Andersen, T. R., Pedersen, E. B. L., Thydén, K. T. S., Helgesen, M., Carlé, J. C., Jørgensen, P. S., Reinhardt, J., Søndergaard, R. R., Jørgensen, M., Bundgaard, E., Krebs, F. C., and Andreasen, J. W., "Enabling flexible polymer tandem solar cells by 3D ptychographic imaging," Advanced Energy Materials 1400736 (2014).

[29] Lai, B., Stankevic, T., Dzhigaev, D., Bi, Z., Rose, M., Shabalin, A., Reinhardt, J., Mikkelsen, A., Samuelson, L., Falkenberg, G., Vartaniants, I., and Feidenhans'l, R., "Nanofocused x-ray beams applied for mapping strain in core-shell nanowires," Proc. SPIE 9592, 95920D (2015).

[30] Dzhigaev, D., Shabalin, A., Stankevic, T., Lorenz, U., Kurta, R. P., Seiboth, F., Wallentin, J., Singer, A., Lazarev, S., Yefanov, O. M., Borgström, M., Strikhanov, M. N., Samuelson, L., Falkenberg, G., Schroer, C. G., Mikkelsen, A., Feidenhans'l, R., and Vartanyants, I. A., "Bragg coherent x-ray diffractive imaging of a single indium phosphide nanowire," J. Opt. 18(6), 064007 (2016).

[31] Krause, T., Hanke, M., Nicolai, L., Cheng, Z., Niehle, M., Trampert, A., Kahnt, M., Falkenberg, G., Schroer, C. G., Hartmann, J., Zhou, H., Wehmann, H.-H., and Waag, A., "Structure and composition of isolated core-shell (In,Ga)N/GaN rods based on nanofocus x-ray diffraction and scanning transmission electron microscopy," Phys. Rev. Applied 7(2), 024033 (2017).

[32] Reinhardt, J., Hoppe, R., Hofmann, G., Damsgaard, C. D., Patommel, J., Baumbach, C., Baier, S., Rochet, A., Grunwaldt, J.-D., Falkenberg, G., and Schroer, C. G., "Beamstop-based low-background ptychography to image weakly scattering objects," Ultramicroscopy 173, 52-57 (2017).

[33] Hoppe, R., Reinhardt, J., Hofmann, G., Patommel, J., Grunwaldt, J.-D., Damsgaard, C. D., Wellenreuther, G., Falkenberg, G., and Schroer, C. G., "High-resolution chemical imaging of gold nanoparticles using hard x-ray ptychography," Appl. Phys. Lett. 102(20), 203104 (2013).

[34] Schroer, C. G. and Lengeler, B., "Focusing hard x rays to nanometer dimensions by adiabatically focusing lenses," Phys. Rev. Lett. 94, 054802 (2005).

[35] Patommel, J., Klare, S., Hoppe, R., Ritter, S., Samberg, D., Wittwer, F., Jahn, A., Richter, K., Wenzel, C., Bartha, J. W., Scholz, M., Seiboth, F., Boesenberg, U., Falkenberg, G., and Schroer, C. G., "Focusing hard x rays beyond the critical angle of total reflection by adiabatically focusing lenses," Appl. Phys. Lett. 110(10), 101103 (2017).

[36] Seiboth, F., Scholz, M., Patommel, J., Hoppe, R., Wittwer, F., Reinhardt, J., Seidel, J., Knaut, M., Jahn, A., Richter, K., Bartha, J. W., Falkenberg, G., and Schroer, C. G., "Hard x-ray nanofocusing by refractive lenses of constant thickness," Appl. Phys. Lett. 105(13), 131110 (2014).

[37] Schroer, C. G., Hönig, S., Goldschmidt, A., Hoppe, R., Patommel, J., Samberg, D., Schropp, A., Seiboth, F., Stephan, S., Schöder, S., Burghammer, M., Denecke, M., Wellenreuther, G., and Falkenberg, G., "Hard x-ray nano-beam characterization by ptychographic imaging," Proc. SPIE 8141, 814103 (2011).

[38] Seiboth, F., Kahnt, M., Scholz, M., Seyrich, M., Wittwer, F., Garrevoet, J., Falkenberg, G., Schropp, A., and Schroer, C. G., "Quantitative characterization of aberrations in x-ray optics," Proc. SPIE 9963, 99630P (2016).

[39] Seiboth, F., Schropp, A., Scholz, M., Wittwer, F., Rödel, C., Wünsche, M., Ullsperger, T., Nolte, S., Rahomäki, J., Parfeniukas, K., Giakoumidis, S., Vogt, U., Wagner, U., Rau, C., Boesenberg, U., Garrevoet, J., Falkenberg, G., Galtier, E. C., Lee, H. J., Nagler, B., and Schroer, C. G., "Perfect X-ray focusing via fitting corrective glasses to aberrated optics," Nature Communications 8, 14623 (2017). doi: 10.1038/ncomms14623.

[40] Norbert Lindlein and Gerd Leuchs, "Geometrical optics," in [Springer Handbook of Lasers and Optics], Träger, F., ed., ch. 2, 35-88, Springer, 2nd ed. (2012).

[41] Schropp, A., Hoppe, R., Meier, V., Patommel, J., Seiboth, F., Lee, H. J., Nagler, B., Galtier, E. C., Arnold, B., Zastrau, U., Hastings, J. B., Nilsson, D., Uhlen, F., Vogt, U., Hertz, H. M., and Schroer, C. G., "Full spatial characterization of a nanofocused x-ray free-electron laser beam by ptychographic imaging," Scientific Reports 3, 1633 (2013). 